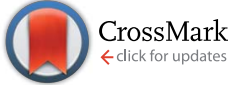

Cite this: J. Mater. Chem. A, 2015, 3 , 5420

Received 3rd December 2014 Accepted 15th January 2015

DOI: $10.1039 / c 4 t a 06642 a$

www.rsc.org/MaterialsA

\section{Highly branched cobalt phosphide nanostructures for hydrogen-evolution electrocatalysis $\uparrow$}

\begin{abstract}
Eric J. Popczun, ${ }^{a}$ Christopher W. Roske, ${ }^{b}$ Carlos G. Read, ${ }^{a}$ J. Chance Crompton, ${ }^{b}$ Joshua M. McEnaney, ${ }^{a}$ Juan F. Callejas, ${ }^{a}$ Nathan S. Lewis ${ }^{* b}$ and Raymond E. Schaak ${ }^{* a}$

CoP nanostructures that exposed predominantly (111) crystal facets were synthesized and evaluated for performance as electrocatalysts for the hydrogen-evolution reaction (HER). The branched CoP nanostructures were synthesized by reacting cobalt(II) acetylacetonate with trioctylphosphine in the presence of trioctylphosphine oxide. Electrodes comprised of the branched CoP nanostructures deposited at a loading density of $\sim 1 \mathrm{mg} \mathrm{cm}^{-2}$ on Ti electrodes required an overpotential of $-117 \mathrm{mV}$ to produce a current density of $-20 \mathrm{~mA} \mathrm{~cm}^{-2}$ in $0.50 \mathrm{M} \mathrm{H}_{2} \mathrm{SO}_{4}$. Hence the branched CoP nanostructures belong to the growing family of highly active non-noble-metal HER electrocatalysts. Comparisons with related CoP systems have provided insights into the impact that shape-controlled nanoparticles and nanoparticle-electrode interactions have on the activity and stability of nanostructured HER electrocatalysts.
\end{abstract}

\section{Introduction}

Transition metal phosphides have recently emerged as an important family of highly active electrocatalysts that facilitate the production of molecular hydrogen from acidic aqueous solutions, which is important for clean-energy technologies such as water electrolyzers and solar fuels generators. For example, phosphides of nickel, ${ }^{1-3}$ cobalt, ${ }^{4-12}$ iron, ${ }^{13,14}$ copper, ${ }^{15}$ molybdenum, ${ }^{\mathbf{1 6 - 1 8}}$ and tungsten ${ }^{19}$ have been found to electrocatalytically generate $\mathrm{H}_{2}(\mathrm{~g})$ with low overpotentials at operationally relevant current densities for solar-driven water splitting systems, while exhibiting high stability under strongly acidic conditions. Metal phosphides therefore offer an earthabundant and inexpensive alternative to platinum, which serves as the benchmark catalyst for the hydrogen-evolution reaction (HER).

The relationship between the electrocatalytic activity and the exposed crystal face has not yet been elucidated for the transition metal phosphides, which are generally studied as nanocrystals. The (001) surface of $\mathrm{Ni}_{2} \mathrm{P}$ contains proximate phosphorus and nickel sites that are hypothesized to work cooperatively to facilitate moderate binding of the intermediates and products to the catalyst surface. ${ }^{20}$ The other metal phosphides that have been identified as HER catalysts have

\footnotetext{
${ }^{a}$ Department of Chemistry and Materials Research Institute, The Pennsylvania State University, University Park, PA 16802, USA. E-mail: res20@psu.edu

${ }^{b}$ Division of Chemistry and Chemical Engineering, California Institute of Technology, Pasadena, CA 91125, USA. E-mail: nslewis@caltech.edu

$\dagger$ Electronic supplementary information (ESI) available: Additional experimental details, calculations, materials characterization, and electrochemical data. See DOI: $10.1039 / \mathrm{c} 4 \mathrm{ta} 06642 \mathrm{a}$
}

related crystal structures which also contain proximate metal and phosphorus structural motifs on their low-index surfaces. This structural feature suggests that metal phosphide nanostructures that preferentially expose different crystal facets would be expected to exhibit different activities for HER catalysis.

The CoP system, which has been studied extensively, exhibits high HER activity across a diverse group of morphologies, characteristic grain sizes, support materials, and synthetic preparations. For example, nominally comparable activity has been observed for multi-faceted single-crystalline hollow CoP nanoparticles on $\mathrm{Ti}$ foil, ${ }^{\mathbf{4}}$ electrochemically deposited CoP films, ${ }^{9}$ CoP nanocrystals on carbon nanotube ${ }^{7}$ and carbon cloth supports, ${ }^{10}$ porous CoP nanowires on carbon cloth $^{5}$ and $\mathrm{Ti}$ substrates, ${ }^{6}$ CoP nanosheets on a Ti plate, ${ }^{8}$ porous templategrown CoP nanowires, ${ }^{11}$ and a collection of morphologically variant CoP nanoparticles on glassy carbon electrodes. ${ }^{6}$ The primary methods used to synthesize these CoP nanostructures include reaction of pre-made Co nanoparticles with trioctylphosphine, or phosphidation of cobalt oxide and related nanostructured templates. ${ }^{4-8}$ Although these results collectively demonstrate the high inherent HER activity of CoP nanostructures, a direct comparison of the activities and performance is difficult for samples made by different methods and for electrodes prepared in different laboratories, because of unavoidable differences in the electrode preparation methods, mass loadings, and accessible surface areas. Additionally, the HER-active CoP nanostructures reported to date have been polycrystalline or multi-faceted, without statistically relevant differences in the proportions of the different crystal facets that have been exposed and accessible. 
An important first step toward the goal of better understanding the origin of the high HER activity in CoP and related nanostructured metal phosphide systems is to synthesize and study high quality, morphologically distinct samples. CoP is ideally suited for such an approach, because multi-faceted single-crystalline CoP nanoparticles have amongst the highest activities and acid stabilities of the metal phosphide family of HER catalysts. ${ }^{4}$ Also, using closely related chemical strategies, methods exist for synthesizing CoP and related metal phosphide nanostructures to produce materials of the same chemical composition and structure but with different morphologies. Accordingly, we report herein the synthesis of highly branched CoP nanostructures with single-crystal CoP nanorod protrusions that expose a high density of accessible (111) facets. The electrocatalytic HER performance of the branched CoP nanostructures was evaluated and compared to that of multi-faceted CoP nanocrystals. ${ }^{4}$ These observations provide a starting point for the identification of the key parameters that impact the HER performance of morphologically distinct nanostructures in the same materials system.

\section{Experimental}

\section{Chemicals and materials}

1-Octadecene [tech. 90\%, $\mathrm{C}_{18} \mathrm{H}_{36}$ ], oleylamine [70\%, $\mathrm{C}_{18} \mathrm{H}_{37} \mathrm{~N}$ ], trioctylphosphine [TOP, 97\%, $\left.\left(\mathrm{C}_{8} \mathrm{H}_{17}\right)_{3} \mathrm{P}\right]$, trioctylphosphine oxide [TOPO, 99\%, $\left(\mathrm{C}_{8} \mathrm{H}_{17}\right)_{3} \mathrm{PO}$ ], titanium foil [99.7\%, $0.25 \mathrm{~mm}$ thickness], and sulfuric acid [99.999\%] were purchased from Sigma-Aldrich, and cobalt(II) acetylacetonate $\left[\mathrm{Co}(\mathrm{acac})_{2}\right]$ was purchased from Alfa Aesar. All chemicals were used as received. Silver paint was purchased from SPI supplies, and two-part epoxy [HYSOL 1C] was purchased from McMaster-Carr.

\section{Synthesis of highly branched CoP nanostructures}

$\mathrm{Co}(\mathrm{acac})_{2}(256 \mathrm{mg}, 1.0 \mathrm{mmol})$ was introduced into a threenecked round bottom flask that contained oleylamine $(6.4 \mathrm{~mL}$, $19.4 \mathrm{mmol})$, TOPO (3.8 g, $9.8 \mathrm{mmol})$, TOP (0.5 mL, $1.1 \mathrm{mmol})$, and a magnetic borosilicate-coated stir bar. The flask was placed into a heating mantle and was equipped with a mercury thermometer with a thermometer adapter in the first port, a Liebig condenser with gas inlet adapter in the second port, and a glass pennywise stopper in the final port. The reaction mixture was heated to $120^{\circ} \mathrm{C}$ for $1 \mathrm{~h}$ under vacuum to remove water and other low-boiling impurities. Following degassing, an $\operatorname{Ar}(\mathrm{g})$ blanket was introduced to the reaction flask while the temperature was raised to $355-360{ }^{\circ} \mathrm{C}$ and then maintained for $3 \mathrm{~h}$. Following heating, the reaction flask was cooled rapidly by removing the heating mantle. Upon reaching $80^{\circ} \mathrm{C}$, the flask was opened to the atmosphere and $\sim 10 \mathrm{~mL}$ of toluene was added to the reaction to avoid solidification of the TOPO. To isolate the nanoparticles, the reaction solution was separated into centrifuge tubes, with each tube being $~ 1 / 3$ full. An equal volume of isopropyl alcohol was added to the centrifuge tubes to promote precipitation. The tubes were sealed and then centrifuged at $7000 \mathrm{rpm}$ for $3 \mathrm{~min}$. The resulting supernatant was discarded and the particles were resuspended by addition of hexanes. The particles were then flocculated by addition of excess isopropyl alcohol and centrifuged again. This entire process was repeated two additional times. The resulting oily residue that contained the nanoparticle precipitate was resuspended in $0.50 \mathrm{M} \mathrm{H}_{2} \mathrm{SO}_{4}$ and centrifuged to collect the final $\mathrm{CoP}$ powder, which was suspended in hexanes for storage.

\section{Preparation of working electrodes}

Working electrodes were prepared by first making $10 \mathrm{~mL}$ of a CoP nanostructure stock solution in hexanes $\left(10 \mathrm{mg} \mathrm{mL}^{-1}\right)$. To this suspension, $\sim 5 \mu \mathrm{L}$ of oleylamine was added to help promote particle adhesion to the electrode surface. To a $0.2 \mathrm{~cm}^{2}$ piece of titanium foil, $20 \mu \mathrm{L}$ of the CoP nanostructure stock solution was deposited in $5 \mu \mathrm{L}$ increments to achieve a loading density of $\sim 1 \mathrm{mg} \mathrm{cm}^{-2}$. The resulting CoP-decorated Ti foils were annealed under $\mathrm{H}_{2}(5 \%) / \mathrm{Ar}(95 \%)$ at $450{ }^{\circ} \mathrm{C}$ for $30 \mathrm{~min}$. The loading densities were validated experimentally using a microbalance, by taking the difference between the mass of the annealed foils and the mass of the initial foil. The back sides of the nanostructure-loaded Ti foils were attached to polyvinylchloride-coated $\mathrm{Cu}$ wires with $\mathrm{Ag}$ paint. The wires were placed through a $6 \mathrm{~mm}$-diameter glass capillary and two-part epoxy was used to cover all exposed surfaces except the front of the Ti foil, onto which the CoP nanostructure sample was deposited.

\section{Electrochemical characterization}

Electrochemical data were collected using a Gamry Instruments Reference 600 potentiostat. All measurements were performed in $0.50 \mathrm{M} \mathrm{H}_{2} \mathrm{SO}_{4}$, unless otherwise noted. A single-compartment cell was used with a graphite rod counter electrode and mercury-mercury sulfate $\left(\mathrm{Hg} / \mathrm{Hg}_{2} \mathrm{SO}_{4}\right)$ reference electrode. Polarization data were collected at a scan rate of $2 \mathrm{mV} \mathrm{s}^{-1}$, with the solution agitated using a magnetic stir bar. The data were corrected for uncompensated resistance ( $\sim 6$ ohms) using the current-interrupt method that was built into the Reference 600 potentiostat software suite. The reversible hydrogen electrode (RHE) potential was maintained by continuous bubbling of $\sim 1$ atm of research-grade $\mathrm{H}_{2}(\mathrm{~g})$ into the electrochemical cell. To determine the RHE potential, the open-circuit potential of a platinum mesh electrode was determined following the completion of any experiments involving CoP, to avoid $\mathrm{Pt}$ contamination. To test the short-term stability, the potential was held at a current density of $-10 \mathrm{~mA} \mathrm{~cm}^{-2}$ for $18 \mathrm{~h}$ of continuous galvanostatic testing. To test the long-term stability, accelerated degradation studies were performed using cyclic voltammetry from $+5 \mathrm{mV}$ to $-160 \mathrm{mV} v s$. RHE for 500 cycles at a scan rate of $100 \mathrm{mV} \mathrm{s}^{-1}$.

\section{Materials characterization}

Transmission-electron microscopy (TEM) images were collected using a JEOL 1200 microscope operating at an accelerating voltage of $80 \mathrm{kV}$. A JEOL JEM-2010F was used to collect highresolution bright-field TEM images as well as to obtain energydispersive X-ray spectroscopy (EDX) data at an accelerating voltage of $200 \mathrm{kV}$. Gatan Digital Micrograph software was used to measure the lattice spacings from the fast-Fourier transform 
(FFT) of the HRTEM images. These values, and the corresponding FFT diffraction patterns, were simulated using the CrystalMaker/SingleCrystal software package. Scanning-electron microscopy (SEM) images were collected using a FEI Nova NanoSEM 630 at a working distance of $1.5 \mathrm{~mm}$ and an accelerating voltage of $2.00 \mathrm{kV}$. Powder X-ray diffraction (XRD) patterns were collected using a Bruker-AXS D8 Advance diffractometer with $\mathrm{Cu} \mathrm{K} \alpha$ radiation and a LynxEye 1-D detector operating at room temperature. The CrystalMaker/CrystalDiffract software package was also used to simulate the XRD patterns, using previously published crystallographic parameters for MnP-type CoP. ${ }^{21}$ Brunauer-Emmett-Teller (BET) surface area measurements were performed using a Micromeritics ASAP 2020 at liquid-nitrogen temperatures, and the data were analyzed using the ASAP 2020 software version 4.0.

\section{Results and discussion}

Fig. 1 shows TEM images for the highly branched CoP nanostructures that formed upon heating $\mathrm{Co}(\mathrm{acac})_{2}$, TOP, TOPO, and oleylamine at $360{ }^{\circ} \mathrm{C}$ for $3 \mathrm{~h}$. A majority of the CoP nanostructures consisted of a central core with multiple nanorod protrusions that grew outward. The nanorod protrusions had an average diameter of $14 \pm 3 \mathrm{~nm}$ and the average diameter of the
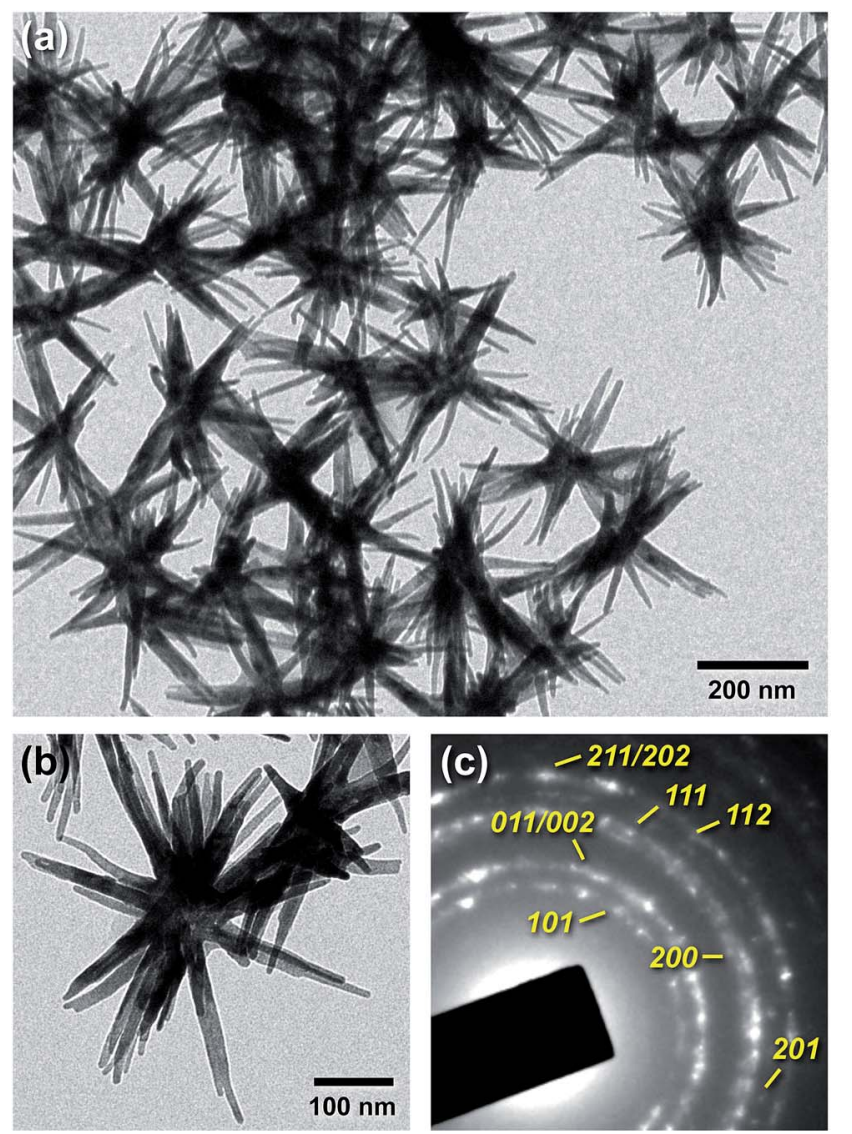

Fig. 1 ( $a$ and b) TEM images and (c) corresponding SAED pattern of a representative sample of highly branched CoP nanostructures. The indexing in (c) corresponds to MnP-type CoP. complete nanostructures was $390 \pm 70 \mathrm{~nm}$. The SEM image in Fig. 2 confirmed the three-dimensional branched morphology of the CoP nanostructures, as well as their uniformity. HRTEM images of both the core (Fig. 3a) and the branches (Fig. 3b-d) indicated that both regions were crystalline and that the branches appeared to be single-crystalline. The majority of the nanorods that protruded from the core predominantly exposed the (111) lattice plane of CoP, with a lattice spacing of $2.4 \AA$.

Fig. 4 shows powder XRD data for a bulk sample of the CoP branched nanostructures, with a SAED pattern and an EDS spectrum that both correspond to the TEM image shown in Fig. 1a included in Fig. 1c and $\mathrm{S} 1, \dagger$ respectively. The experimental powder XRD pattern in Fig. 4 matches well with that expected for MnP-type CoP, with no observable crystalline impurities. The agreement between the relative peak intensities that were observed experimentally $v s$. those that are expected for an isotropic sample indicates that no significant preferred orientation was present in the sample. Strong preferred orientation would be expected for nanorods, but not for the branched nanostructures with three-dimensionally protruding nanorods, which cannot align along a preferential crystal direction upon deposition onto a flat substrate. The lack of preferred orientation in the bulk powder XRD sample is therefore consistent with the morphology observed by TEM and SEM. Scherrer analysis of the peak widths indicated an average grain size of $17 \mathrm{~nm}$, which is, within expected experimental error, consistent with the average diameter of the single-crystalline nanorod protrusions as observed by TEM.

The SAED pattern in Fig. 1c is also consistent with MnP-type $\mathrm{CoP}$, and therefore matches well with that observed for the bulk sample by powder XRD. The EDS spectrum in Fig. S1, $\uparrow$ acquired for an ensemble of particles, indicated a Co : P ratio of $50: 50$, which matches with that expected for CoP. Taken together, the XRD, SAED, and EDS data therefore confirm the assigned composition and crystal phase, and indicate that the CoP branched nanostructures formed with high purity.

Although highly branched colloidal CoP nanostructures apparently have not been synthesized previously, morphologically

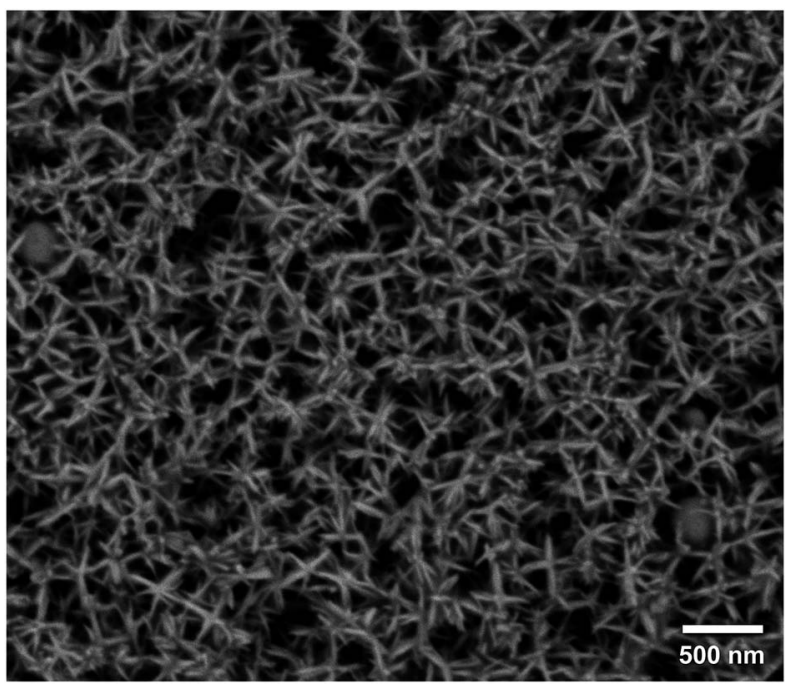

Fig. 2 SEM image of a sample of highly branched CoP nanostructures. 

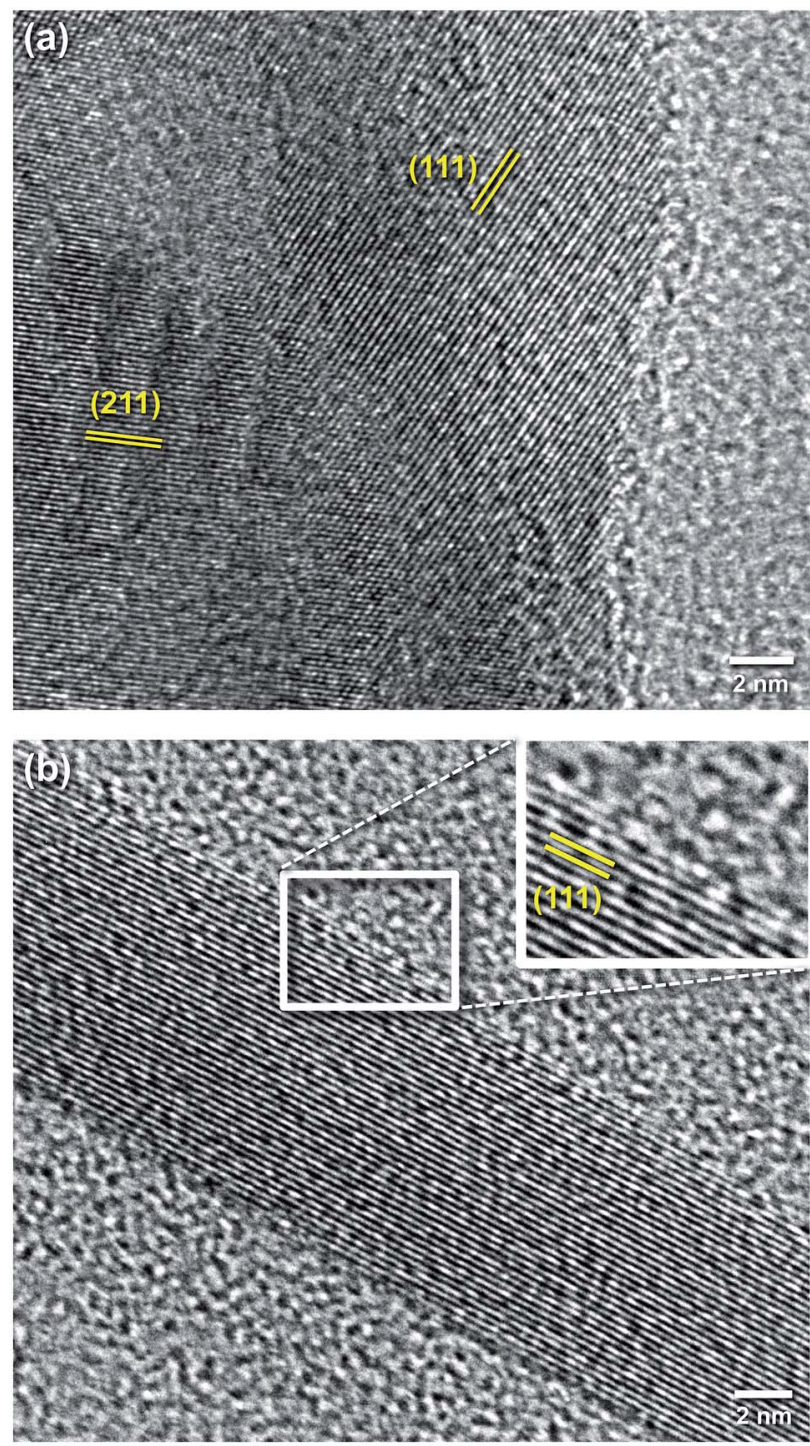

Fig. 3 HRTEM images of (a) the core region and (b) a nanorod branch of a representative CoP nanostructure.

similar $\mathrm{Co}_{2} \mathrm{P}$ nanostructures have been reported. ${ }^{22}$ To access highly branched $\mathrm{Co}_{2} \mathrm{P}$ nanostructures, Zhang and Robinson thermally decomposed cobalt(II) oleate in the presence of pure TOPO, which was the lone phosphorus source. ${ }^{22}$ Our protocol for synthesizing highly branched CoP nanostructures also used TOPO, but we observed that TOPO alone did not produce CoP. Instead, OLAM and TOP were required to form CoP, suggesting that TOP is the primary phosphorus source under our conditions. TOPO is required to generate the highly branched CoP nanostructures, however, rather than the morphologically distinct pseudo-spherical particles that formed when only TOP was used. When TOPO was used as a co-solvent, the highly branched CoP nanostructures form reproducibly, suggesting that TOPO is involved in directing the growth of the nanorod protrusions and/ or stabilizing the predominantly exposed facets.

The branched CoP nanostructures were highly crystalline and exposed predominantly single-crystal (111) facets, as compared to the multi-faceted CoP nanoparticles that have

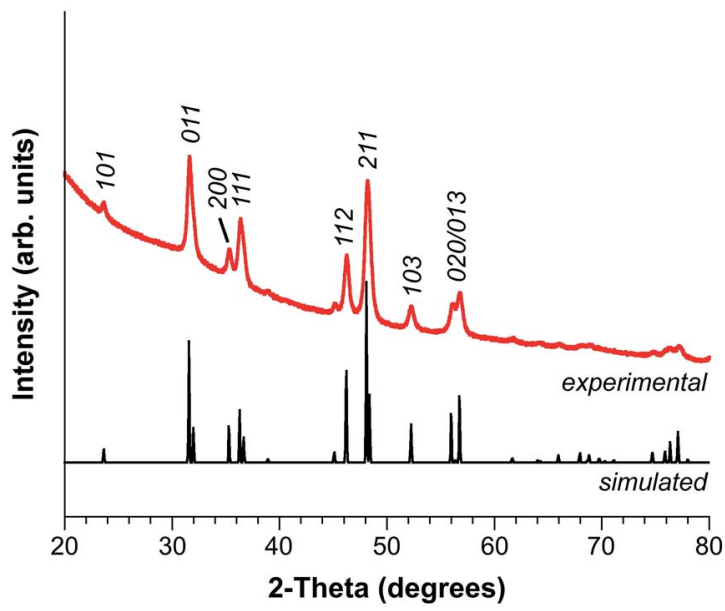

Fig. 4 Powder XRD pattern for the as-synthesized CoP nanostructures. The simulated XRD pattern for MnP-type CoP is shown for comparison.

been synthesized previously. ${ }^{4}$ Furthermore, the surface areas, as measured by Brunauer, Emmett, and Teller (BET) analysis, are comparable for the two different morphologies, with the branched CoP nanostructures having a BET specific surface area of $66 \mathrm{~m}^{2} \mathrm{~g}^{-1}$ and the multi-faceted CoP nanoparticles having a BET specific surface area of $59 \mathrm{~m}^{2} \mathrm{~g}^{-1}$. $^{4}$

The similar BET areas for the two different CoP nanostructures provides an opportunity to evaluate the role of morphology on the HER performance of an earth-abundant HER electrocatalyst. Hence electrodes coated with the branched CoP nanostructures were prepared in an analogous manner to the multi-faceted CoP nanoparticles studied previously. ${ }^{4}$ Briefly, the highly branched CoP nanostructures were deposited onto $\sim 0.2 \mathrm{~cm}^{2}$ Ti substrates from a hexanes dispersion that contained $\sim 0.1$ vol\% oleylamine. The mass loadings were $\sim 1 \mathrm{mg}$ $\mathrm{cm}^{-2}$, and the electrodes were annealed under $\mathrm{H}_{2}(5 \%) / \operatorname{Ar}(95 \%)$ to remove the surface ligands. The SEM and XRD data in Fig. S2† indicate that both the morphology and crystal phase of the CoP nanostructures remained largely unchanged during the electrode preparation and annealing steps, although a small $\mathrm{Co}_{2} \mathrm{P}$ impurity was observed. The Ti electrode containing the branched CoP nanostructures exposed a high density of accessible (111) lattice planes, as shown schematically in Fig. 5.

Fig. 6 shows the polarization (current density vs. potential, $J-E$ ) data that correspond to the HER activity in $0.50 \mathrm{M} \mathrm{H}_{2} \mathrm{SO}_{4}$ of the highly branched CoP nanostructures. The HER activity of the branched CoP nanostructures was lower than that of the multi-faceted CoP nanoparticles reported previously (Fig. S3†), ${ }^{4}$ despite the mutually comparable surface areas for the two different CoP electrocatalyst morphologies. The overpotentials required to produce a (cathodic) current density of $-20 \mathrm{~mA}$ $\mathrm{cm}^{-2}$ were $-117 \mathrm{mV}$ and $-100 \mathrm{mV}$ for the branched nanostructures and multi-faceted nanoparticles, ${ }^{\mathbf{4}}$ respectively, despite their similar loading densities on analogous $\mathrm{Ti}$ substrates. Additionally, the material outperforms previously reported $\mathrm{Co}_{2} \mathrm{P}$ nanostructures $\left(\eta_{-20 \mathrm{~mA} \mathrm{~cm}}{ }^{-2}=-167 \mathrm{mV}\right){ }^{12}$ suggesting that the small $\mathrm{Co}_{2} \mathrm{P}$ impurity does not play a 


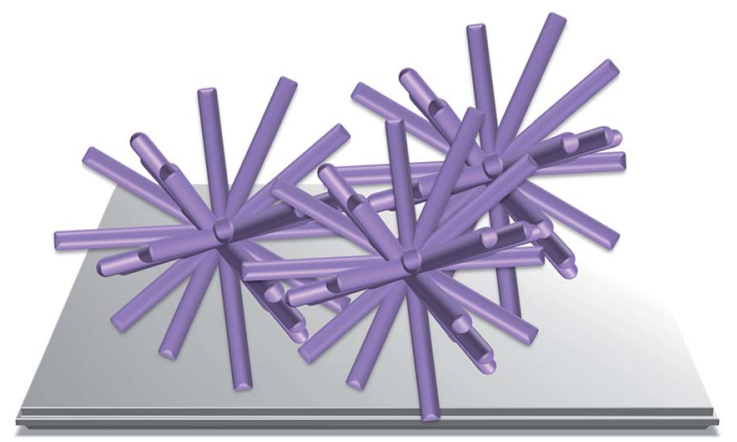

Fig. 5 Schematic highlighting the high density of exposed (111) facets on the $\mathrm{Ti}$ electrodes that contain the highly branched CoP nanostructures.

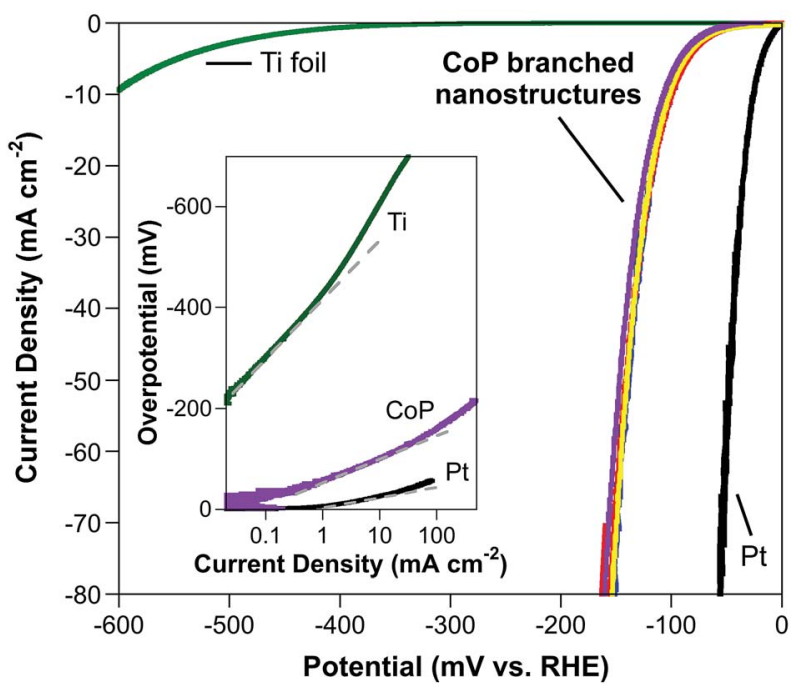

Fig. 6 Polarization data in $0.50 \mathrm{M} \mathrm{H}_{2} \mathrm{SO}_{4}$ for 6 distinct electrodes of the branched CoP nanostructures on Ti foil substrates at mass loadings of $\sim 1 \mathrm{mg} \mathrm{cm}^{-2}$, along with $\mathrm{Ti}$ foil and $\mathrm{Pt}$ mesh electrodes for comparison. Inset: Tafel plots for the Ti and Pt electrodes, as well as a representative branched CoP/Ti electrode. Tafel slopes, denoted by the grey dashed lines, were calculated using the following linear regions: $-60 \mathrm{mV}>\eta>-125 \mathrm{mV}$ for $\mathrm{CoP},-10 \mathrm{mV}>\eta>-60 \mathrm{mV}$ for Pt, and $-250 \mathrm{mV}>\eta>-400 \mathrm{mV}$ for $\mathrm{Ti}$.

significant role in the observed activity of the branched CoP nanorods. A quantitative Faradaic yield of $\mathrm{H}_{2}(\mathrm{~g})$ was observed for both types of CoP catalytic systems (see ESI $\dagger$ ). A Tafel analysis, shown in the inset to Fig. 6, revealed Tafel slopes of $29 \mathrm{mV}$ per decade and $123 \mathrm{mV}$ per decade for the Pt mesh and Ti foil controls. Both of these values are consistent with values expected based on literature reports. ${ }^{1,4,23}$ The Tafel slope for the branched CoP nanostructures was $48 \mathrm{mV}$ per decade, which is comparable to the $50 \mathrm{mV}$ per decade Tafel slope observed previously for the multi-faceted pseudo-spherical CoP nanoparticles. ${ }^{4}$ The comparable values suggest a similar mechanism for the HER on the branched CoP nanostructures and on the CoP nanoparticles.

Given the different activities of the branched CoP nanostructures $v s$. the CoP nanoparticles, the turnover frequency (TOF) values are necessarily quite different (see the ESI $\uparrow$ for TOF calculations). The branched CoP nanostructures exhibited a TOF of $0.019 \mathrm{~s}^{-1}$ at an overpotential $(\eta)$ of $-100 \mathrm{mV}$. This TOF value is lower than that of CoP nanoparticles, which showed a TOF $=$ $0.060 \mathrm{~s}^{-1}$ at $\eta=-100 \mathrm{mV}^{4}$ The branched CoP nanostructures, however, still provided exceptionally high activity for the HER, performing comparably to many other non-noble-metal HER catalysts in acidic solutions, based on evaluation metrics reported in the literature. Examples include $\operatorname{MoS}_{2}\left(\eta_{-20 \mathrm{~mA} \mathrm{~cm}}{ }^{-2}=\right.$ $-175 \mathrm{mV}),{ }^{24} \mathrm{Mo}_{2} \mathrm{C}\left(\eta_{-10 \mathrm{~mA} \mathrm{~cm}}{ }^{-2}=-152 \mathrm{mV}\right),{ }^{25} \mathrm{CoS}_{2}\left(\eta_{-10 \mathrm{~mA} \mathrm{~cm}}{ }^{-2}\right.$ $=-145 \mathrm{mV}),{ }^{26} \operatorname{CoSe}_{2}\left(\eta_{-20} \mathrm{~mA} \mathrm{~cm}{ }^{-2}=\sim-155 \mathrm{mV}\right),{ }^{27} \mathrm{MoP}$ $\left(\eta_{-20 \mathrm{~mA} \mathrm{~cm}^{-2}}=-110 \mathrm{mV}\right),{ }^{17} \mathrm{WP}\left(\eta_{-20 \mathrm{~mA} \mathrm{~cm}^{-2}}=-140 \mathrm{mV}\right)^{19}$ and $\mathrm{Ni}_{2} \mathrm{P}\left(\eta_{-20 \mathrm{~mA} \mathrm{~cm}-2}=-130 \mathrm{mV}\right){ }^{1}$ However, the long-term stability of the branched CoP nanostructures on a Ti electrode was inferior to that of the CoP nanoparticles, presumably due to poor adhesion of the branched CoP nanostructures to the Ti electrode surface. ${ }^{4}$ Specifically, under galvanostatic conditions (holding at $-10 \mathrm{~mA} \mathrm{~cm}{ }^{-2}$ for $18 \mathrm{~h}$ ), the particles physically detached from the electrode surface, and the overpotential increased from $-113 \mathrm{mV}$ to $-174 \mathrm{mV}$ over this time period (Fig. S4a $\uparrow$ ). Likewise, 500 cycles between $-160 \mathrm{mV}$ and $+5 \mathrm{mV}$ ( $v s$. the reversible hydrogen electrode, RHE), which simulated multiple catalytic start/stop cycles, resulted in an increase in overpotential at $-10 \mathrm{~mA} \mathrm{~cm}{ }^{-2}$ from $-115 \mathrm{mV}$ to $-135 \mathrm{mV}$ (Fig. S4b $\dagger$ ). The instability is therefore attributed to poor adhesion and is not reflective of the behavior of the catalytic CoP nanostructures themselves.

Typically, differences in activities among morphologically distinct nanostructures of the same compound are routinely attributed to inherent differences in the activities of the exposed crystal facets. However, the complexity of such nanostructured materials and their electrodes makes it difficult to unambiguously determine the reason(s) for the inferior performance of the branched CoP nanostructures relative to their nanoparticulate analogues. Indeed, the (111) surface could inherently be less active for the HER than one or more other surfaces that are simultaneously exposed and accessible on the CoP nanoparticles. However, the lower HER activity of the branched CoP nanostructures could also result from poorer adhesion and/or a lower density of direct CoP-Ti contacts on the electrode surface. Despite this issue, the present study is a first step toward evaluating the relative HER activities of distinct nanostructured catalysts of the same material that are of high morphological quality and uniformity. As such, these results begin to place empirical boundaries on the range of overpotentials that are observed for similarly prepared materials and electrodes. The branched CoP nanostructures nevertheless offer exceptionally high HER activity, exceeding that of most other non-noble-metal systems. These results suggest that nanostructuring to expose specific facets may not be necessary to achieve high HER performance. Indeed, comparable HER activity has been observed by several groups for CoP materials that span a range of morphologies, synthetic protocols, accessible surface areas, and support materials..$^{4-11}$ A key finding of this work is that the activities are largely the same, regardless of morphology or preparation method, underscoring the high intrinsic activity of CoP. The intrinsic HER activity of CoP makes it a highly viable candidate for practical applications, regardless 
of morphological details, and this study reinforces that hypothesis. However, establishing trends in activity that can be correlated to specific crystal facets, surface areas, and electrode materials is still important for furthering the understanding of the origin of the HER activity in these systems, and these results represent a step toward this goal.

\section{Conclusions}

Highly branched nanostructures of CoP, with single-crystal nanorod protrusions that predominantly expose (111) facets act as an active HER electrocatalyst. Moreover, the activity of such samples, while not as high as that of comparable multi-faceted pseudospherical CoP nanoparticles, ${ }^{4}$ is still excellent among the growing family of non-noble-metal nanostructured HER electrocatalysts, producing current densities of $-10 \mathrm{~mA} \mathrm{~cm}{ }^{-2}$ and $-20 \mathrm{~mA} \mathrm{~cm}^{-2}$ at overpotentials of $-100 \mathrm{mV}$ and $-117 \mathrm{mV}$, respectively. These results further establish the high intrinsic activity of CoP as an electrocatalyst for the HER and provide important insights into some of the factors that influence its performance, including exposed crystal facets and nanoparticle-electrode interactions.

\section{Acknowledgements}

The work at PSU was supported by the National Science Foundation (NSF) Center for Chemical Innovation on Solar Fuels (CHE-1305124) and at Caltech by the Joint Center for Artificial Photosynthesis, a DOE Energy Innovation Hub, supported through the Office of Science of the U.S. Department of Energy under Award Number DE-SC0004993, as well as the Gordon and Betty Moore Foundation. TEM was performed in the Penn State Microscopy and Cytometry Facility (University Park, PA) and HRTEM, EDS, SEM, and BET data were acquired at the Materials Characterization Laboratory of the Penn State Materials Research Institute. C.W.R. thanks the NSF for a graduate research fellowship.

\section{Notes and references}

1 E. J. Popczun, J. R. McKone, C. G. Read, A. J. Biacchi, A. M. Wiltrout, N. S. Lewis and R. E. Schaak, J. Am. Chem. Soc., 2013, 135, 9267.

2 L. Feng, H. Vrubel, M. Bensimon and X. Hu, Phys. Chem. Chem. Phys., 2014, 16, 5917.

3 Z. Huang, Z. Chen, Z. Chen, C. Lv, H. Meng and C. Zhang, ACS Nano, 2014, 8, 8121.

4 E. J. Popczun, C. G. Read, C. W. Roske, N. S. Lewis and R. E. Schaak, Angew. Chem., Int. Ed., 2014, 53, 5427.

5 J. Tian, Q. Liu, A. M. Asiri and X. Sun, J. Am. Chem. Soc., 2014, 136, 7587.
6 P. Jiang, Q. Liu, C. Ge, W. Cui, Z. Pu, A. M. Asiri and X. Sun, J. Mater. Chem. A, 2014, 2, 14634.

7 Q. Liu, J. Tian, W. Cui, P. Jiang, N. Cheng, A. M. Asiri and X. Sun, Angew. Chem., Int. Ed., 2014, 53, 6710.

8 Z. Pu, Q. Liu, P. Jiang, A. M. Asiri, A. Y. Obaid and X. Sun, Chem. Mater., 2014, 26, 4326.

9 F. H. Saadi, A. I. Carim, E. Verlage, J. C. Hemminger, N. S. Lewis and M. P. Soriaga, J. Phys. Chem. C, 2014, 118, 29294.

10 Q. Li, Z. Xing, A. M. Asiri, P. Jiang and X. Sun, Int. J. Hydrogen Energy, 2014, 39, 16806.

11 H. Du, Q. Liu, N. Cheng, A. M. Asiri, X. Sun and C. M. Li, J. Mater. Chem. A, 2014, 2, 14812.

12 Z. Huang, Z. Chen, Z. Chen, C. Lv, M. G. Humphrey and C. Zhang, Nano Energy, 2014, 9, 373.

13 J. F. Callejas, J. M. McEnaney, C. G. Read, J. C. Crompton, A. J. Biacchi, E. J. Popczun, T. R. Gordon, N. S. Lewis and R. E. Schaak, ACS Nano, 2014, 8, 11101.

14 P. Jiang, Q. Liu, Y. Liang, J. Tian, A. M. Asiri and X. Sun, Angew. Chem., Int. Ed., 2014, 53, 12855.

15 J. Tian, Q. Liu, N. Cheng, A. M. Asiri and X. Sun, Angew. Chem., Int. Ed., 2014, 53, 9577.

16 P. Xiao, M. A. Sk, L. Thia, X. Ge, R. J. Lim, J.-Y. Wang, K. H. Lim and X. Wang, Energy Environ. Sci., 2014, 7, 2624.

17 J. M. McEnaney, J. C. Crompton, J. F. Callejas, E. J. Popczun, A. J. Biacchi, N. S. Lewis and R. E. Schaak, Chem. Mater., 2014, 26, 4826.

18 Z. Xing, Q. Liu, A. M. Asiri and X. Sun, Adv. Mater., 2014, 26, 5702.

19 J. M. McEnaney, J. C. Crompton, J. F. Callejas, E. J. Popczun, C. G. Read, N. S. Lewis and R. E. Schaak, Chem. Commun., 2014, 50, 11026.

20 P. Liu and J. A. Rodriguez, J. Am. Chem. Soc., 2005, 127, 14871.

21 S. Rundqvist, Acta Chem. Scand., 1966, 16, 287.

22 H. Zhang, D.-H. Ha, R. Hovden, L. F. Kourkoutis and R. D. Robinson, Nano Lett., 2011, 11, 188.

23 J. O. M. Bockris and E. C. Potter, J. Electrochem. Soc., 1952, 99, 169.

24 Y. Li, H. Wang, L. Xie, Y. Liang, G. Hong and H. Dai, J. Am. Chem. Soc., 2011, 133, 7296.

25 W.-F. Chen, C.-H. Wang, K. Sasaki, N. Marinkovic, W. Xu, J. T. Muckerman, Y. Zhu and R. R. Adzic, Energy Environ. Sci., 2013, 6, 943.

26 M. S. Faber, R. Dziedzic, M. A. Lukowski, N. S. Kaiser, Q. Ding and S. Jin, J. Am. Chem. Soc., 2014, 136, 10053.

27 D. Kong, H. Wang, Z. Lu and Y. Cui, J. Am. Chem. Soc., 2014, 136, 4897. 